\title{
A prospective evaluation of the Flacker-Kiely One Year Mortality Score and the added value of NT-proBNP
}

\author{
Kjell Krüger*1,2 ${ }^{*}$ Bård R. Kittang ${ }^{1,3}$, Sabine P. Solheim ${ }^{1}$, Kristian Jansen ${ }^{1,4}$ \\ ${ }^{1}$ Department for nursing home medicine, Municipality of Bergen, Norway \\ ${ }^{2}$ SEFAS, University of Bergen, Norway \\ ${ }^{3}$ Department of Clinical Science, University of Bergen, Norway \\ ${ }^{4}$ The Research Unit for General Practice in Bergen, NORCE, Norway
}

Received: August 10, 2020

DOI: $10.5430 /$ jnep.v11n2p13
Accepted: September 30, $2020 \quad$ Online Published: October 18, 2020

URL: https://doi.org/10.5430/jnep.v11n2p13

\begin{abstract}
Objective: Several mortality indices have been constructed to aid clinical decision making in older adults. We aimed to prospectively validate the Flacker-Kiely (FK) mortality index in a Norwegian nursing home cohort, which has not been done before, and explore whether NT-ProBNP could improve its discriminatory power.

Methods: We performed a cohort/mortality study. From November 2017 to July 2018, physicians in all public long-term nursing homes in Bergen, Norway, scored residents according to the original Flacker Kiely index. Mortality data were derived from the Norwegian Cause of Death Registry and NT-ProBNP values were obtained from routinely collected blood chemistry. An alternative FK index using the NT-ProBNP-value as a marker for the presence of heart failure was constructed (FK NT-ProBNP index). The ProBNP cut-off value was selected based on a Cox regression model ("dead/alive 1 year"/" NT-ProBNP (Ng/l)", where the value with the highest Youden index was identified. We judged index performance by using c-statistics.

Results: Both the original FK index and the constructed FK NT-ProBNP index discriminated between risk strata. The FK NT-ProBNP index yielded a C-index of 0,66 compared to 0,62 for the original FK index. Optimal discriminatory power was shown with a NT-ProBNP cut-off value of $1595 \mathrm{Ng} / \mathrm{l}$ as heart failure criterion, and FK NT-ProBNP score 6.6.

Conclusions: The prospective mortality estimation ability of the FK-index was comparable to previous retrospective studies. The inclusion of NT-ProBNP as a heart failure criterion strengthen the discriminatory power and utility of the index, both in clinic and administration.
\end{abstract}

Key Words: Nursing home, Palliative care, Mortality index

\section{INTRODUCTION}

A large number of health-related factors in decline are associated with mortality in nursing homes. ${ }^{[1]}$ The application of standardized, structured records and central reporting in the US, Canada and other countries has resulted in the development of several mortality indices to aid clinical decision making in older adults. ${ }^{[2-4]}$
Research comparing these indices is scarce. In a systematic review, Yourman et al identified two validated non-diseasespecific prognostic indices that predict the absolute risk of all-cause mortality in a nursing home setting; $;{ }^{[5]}$ the revised Minimum Data Set Mortality Rating Index (MMRI-R) and the revised Flacker Kiely (Flacker-R) long-stay index for 1-year mortality. ${ }^{[6-8]}$ In a prospective validation study on

*Correspondence: Kjell Krüger; Email: kjell.kruger@gmail.com; Address: Løvåsen Nursing Home, Løvåsveien 26, 5145 Fyllingsdalen, Norway. 
96 US nursing home residents by Kruse et al., the original Flacker Kiely - and the revised Flacker Kiely -R index were shown to be more reliable mortality risk prediction tools than the MMRI and MMRI-R. ${ }^{[9]}$ The discriminatory ability of these indices is consistent with other indices that commonly drive clinical decisions, such as the CHA2DS2-VASc-score for warfarin therapy and the Framingham risk score for lipid therapy. ${ }^{[10,11]}$

For the Flacker Kiely-R index, discriminatory ability was approximately the same in both the original derivation study by Flacker and Kiely (C-statistic 0.71 ), and the validation study by Kruse et al. (C-statistic 0.72), and no difference was found between the original and revised version. ${ }^{[12]}$ The parameters contributing to the FK score are: functional ability score higher than 4 (which gives partial score of 2.5), weight loss, that is loss of 5 pounds or more in the last 30 days or 10 pounds or more in the last 180 days (2.26), shortness of breath (2.08), swallowing problems (1.81), male sex (1.76), body mass index less than $22 \mathrm{~kg} / \mathrm{m}^{2}(1.75)$, congestive heart failure (1.56), and age $>88$ years (1.48).

Assessing whether the patient has heart failure (HF) is the only FK-parameter related to a specific diagnosis. HF is common in the nursing home population and is an established risk factor for mortality. ${ }^{[13,14]}$ Confirming the presence of $\mathrm{HF}$ in nursing home patients is complicated by overlapping symptoms with other diseases, atypical clinical presentation, and a limited access to diagnostic echocardiography. NTProBNP measurement, an essential tool in the diagnostic HF work-up, is an accessible blood chemistry analysis in Norwegian nursing homes. Plasma concentrations of NT-ProBNP provide important prognostic information, both in patients with and without heart failure, ${ }^{[15]}$ predicting 1 -year mortality in nursing home residents independent of age, gender, and morbidity. ${ }^{[16]}$ In the present study we therefore aimed to conduct a prospective validation of the FK index in a Norwegian nursing home cohort, and to explore whether NT-ProBNP could improve the discriminatory power of the FK index.

\section{MeTHODS}

We performed a cohort/mortality study.

\subsection{Study setting}

The study was conducted in nursing homes in the Municipality of Bergen, the second largest city in Norway. In 2019, Bergen had 283,240 inhabitants and a total of 1,869 long-term beds and 522 short-term beds in 24 public and 13 private nursing homes. ${ }^{[17]}$ The one-year mortality rate was $32 \%$ among long-term residents in Bergen in 2018 (unpublished data), in line with annual mortality rates in nursing homes around the country. ${ }^{[18]}$ Norway has in total 5.1 million inhabitants and about 900 nursing homes.

\subsection{Study population}

From November 2017 until July 2018, physicians in all public long-term nursing homes $(n=24)$ were asked to score residents according to the original FK-index. We obtained informed consent from 305 out of the 600 long-term nursing home residents with available FK-score. Mortality data was derived from the Norwegian Cause of Death Registry one year after the end of the inclusion period. The parameters "dead/alive at one year after score" and "days alive after score" were constructed for the purpose of analysis. The total long-term stay nursing home population $(\mathrm{n}=1,869)$ and the population with informed consent $(n=305)$ were compared regarding relevant characteristics.

\subsection{Determination of NT-ProBNP cut-off-value}

A standard set of blood chemistry analyses including NTProBNP ( $\mathrm{Ng} / \mathrm{l})$ are routinely obtained from all long-term residents in the nursing homes of Bergen, both at admission and subsequently every 6 months. In this study, the last available NT-ProBNP-value prior to death/data extraction was used in our analyses.

An alternative FK-index including the NT-ProBNP-value as a marker of the presence of HF was constructed; the FlackerKiely-NT-ProBNP index (FK NT-ProBNP).

\subsection{Statistical analyses}

JMP 14.0.0 from SAS was used for statistical analysis. To get an impression of the correlation between NT-ProBNP and mortality, we performed a survival analysis for three different NT-ProBNP-strata and a Cox regression analysis, including all FK parameters as categorical variables and (NTProBNP Ng/l)/10 as continuous variable. In an alternative FK NT-ProBNP index, the heart failure question in the original FK-score was replaced by a NT-ProBNP cut-off value. The NT-ProBNP cut-off value was identified by a Cox regression analysis $^{[19]}$ for "dead/alive 1 year"/"NT-ProBNP (Ng/l)", selecting the value with lowest Youden indices (Sensitivity - (1 - Specificity)). One-year mortality per FK score-strata was compared between indices with Pearson chi-square test and Cochrane Arbitrage trend test. ${ }^{[20,21]}$ To evaluate index predictive performance we reported $\mathrm{C}$-indices for Cox regression analyses between test scores and "dead/alive at one year", and analyzed the discriminative strength between score strata in a survival analysis of "days alive after FK-testing" for both index versions (original FK, FK NT-ProBNP).

\subsection{Ethics}

The study was approved by the Regional Ethics Committee (REK vest), 2015/2340/REK Vest. The residents were not 
exposed to interventions with potentially harmful effects.

\section{RESUlts}

Informed consent was obtained from a total of 305 patients.

The mean age was 87 years, and $86 \%$ of the residents were fe-

male. Compared to the total long-term population in Bergen, the study population was older, had a lower BMI, lower proBNP value, higher eGFR values, used fewer regular drugs, but a higher number of on-demand drugs (see Table 1) The mortality rate in the study population one year after FK-score was $46 \%$, whereas the overall yearly mortality rate in 2018 was $32 \%$ among all long-term residents (unpublished data).

Table 1. Different characteristics between study group and total long-term population

\begin{tabular}{lll}
\hline & Total long-term population, N 1869 & Tested long-term population, N 305 \\
\hline Sex, (male\%/female \%) & $32 / 68$ & $30 / 70$ \\
\hline & Mean, SD, 95\% CI & Mean, SD, 95\% CI \\
\hline Age (years) & $84.07,10.4,(83.62-84.52)$ & $87.17,8.72,(86.53-87.81)$ \\
BMI (kg/m $\left.{ }^{2}\right)$ & $23.52,4.57,(23.34-23.71)$ & $22.63,4.83,(22.04-22.21)$ \\
Systolic blood pressure (mmHg) & $130.07,22.07,(129.11-131.02)$ & $126.58 .24 .72,(123.59-129.56)$ \\
Hb (g/dl) & $12.49,1.58,(12.42-12.56)$ & $12.31,1.68,(12.11-12.52)$ \\
ProBNP (Ng/l) & $1,018.79,2,386.58,(896.90-1,140.69)$ & $2,008.56,4,669.05,(1,388.15-2,628.95)$ \\
eGFR (ml/min/1.73m $\left.{ }^{2}\right)$ & $69.54,24.95,(68.41-70.67)$ & $64.97,21.63,(62.33-67.60)$ \\
Number of on-demand drugs & $3.75,2.1,(3.66-3.85)$ & $4.92,2.4,(5.22-0.15)$ \\
Number of regular drugs & $6.69,3.30,(6.55-6.83)$ & $5.98,3.73,(5.50-6.47)$ \\
\hline & $\%$ & $\%$ \\
\hline Cancer & 11 & 13 \\
Diabetes mellitus & 15 & 15 \\
Dementia & 52 & 62 \\
Ischemic heart disease & 14 & 16 \\
Atrial fibrillation & 21 & 23 \\
Suffered stroke & 12 & 11 \\
\hline
\end{tabular}

We found significant differences between different strata of NT-ProBNP as regards time to death (see Figure 1A), with the highest NT-proBNP straum in particular having reduced survival. A Cox regression of the FK index individual param- eters showed that proBNP, male gender, functions of daily living, and shortness of breath were significantly associated with one-year mortality (see Figure 1B).

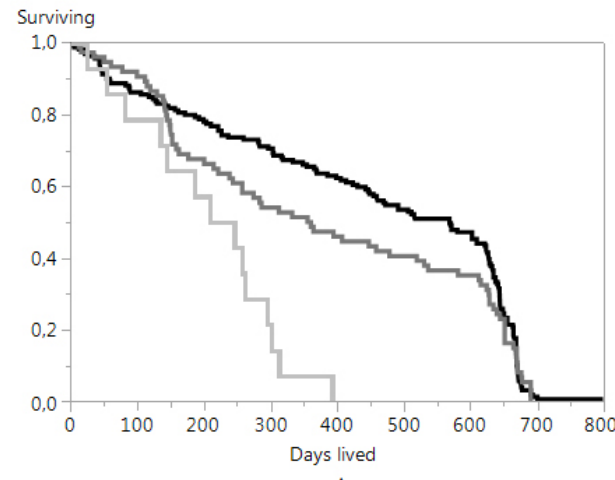

A

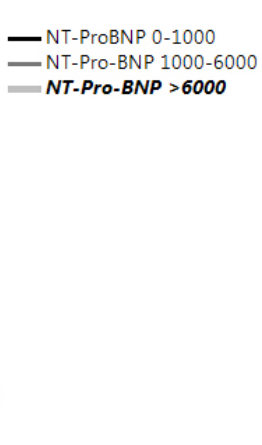

Figure 1. A; Survival of patients by three different strata of NT-ProBNP (Wilcoxon $p<.0001$ ). To test the hypothesis that NT-ProBNP may serve well as heart failure indicator in the actual setting. B; Multiple regression with "Dead/alive 1 year" as dependent variable, comparing contribution of all FK-parameters and NT-ProBNP Ng/l. 
The ROC (Receiver operating characteristic) curve for the Cox regression for NT-ProBNP (Ng/l) and "dead/alive 1 year" gave an Youden index of 0.2684 showing that $1,595 \mathrm{Ng} / \mathrm{l}$ was the most effective cut-off NT-ProBNP value for identifying one-year mortality risk. Hence, this value was used as cut-off constructing the "FK NT-ProBNP index".

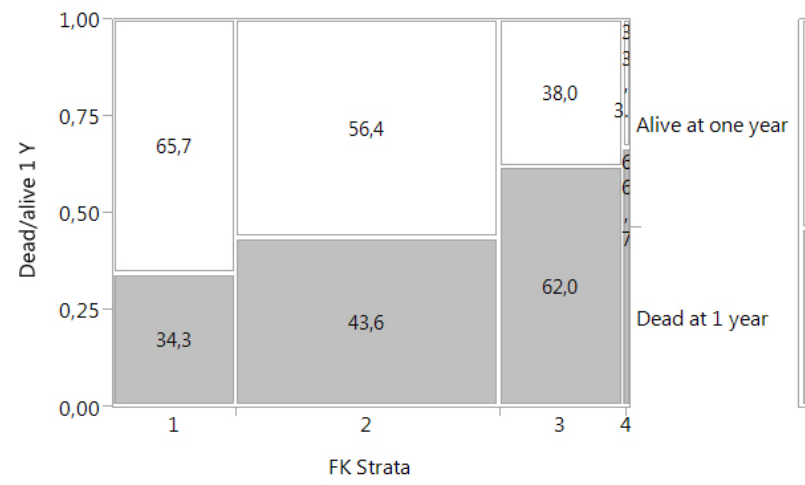

A
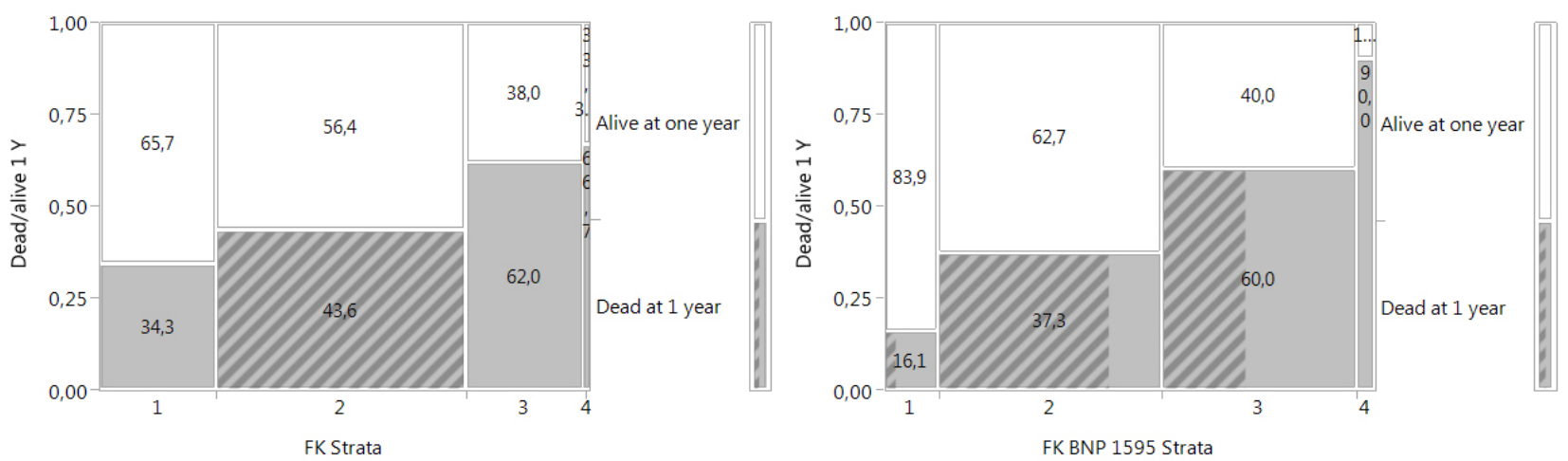

B

Figure 2. A; Proportion of deceased patients per stratum during 12 months for the original Flacker Kiely test (left) and FK NT-ProBNP (right). On the X-axis " 1 " refers to FK-score $>=0<3$, "2" if $>=3<7$, "3" if $>=7<11$ and "4" if $>=11$. B; Demonstrates how patients from stratum 2 (striped field) of the original test (left) are redistributed across strata 1, 2 and 3 of the "FK NT-ProBNP test" (right).
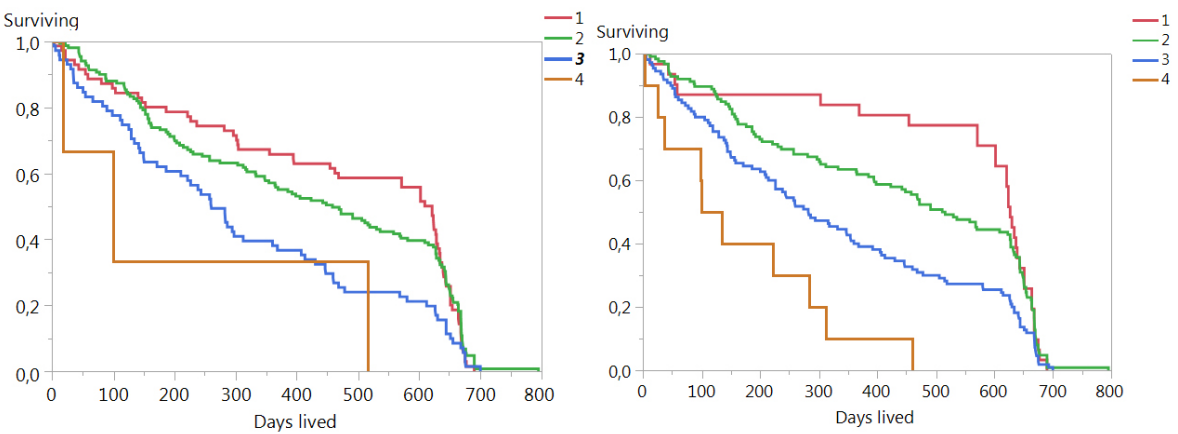

Figure 3. Survival analysis for the time after testing. Left the "FK test" and right "FK NT-ProBNP test". Legend-numbers refer to different score strata, "1" to score $>-0<3$, "2" $>-3<7$, " 3 " $>-7<11$ and " 4 " $11+$ 
The FK-index values most effectively predicting 1-year mortality based on the Youden index of the Cox regression analysis with the variable "dead/alive 1 year" for the two test variants, (FK, FK NT-ProBNP) were 4.8 and 6.6, respectively. A cut-off value for NT-ProBNP of $1,595 \mathrm{Ng} / \mathrm{l}$ and a FK NT-ProBNP score of 6.6 gave the highest C-index and thus the best discriminatory power for one-year mortality risk, with a C-index of 0.66 compared to 0.62 for the original test (see Figure 3).

\section{Discussion}

To our best knowledge, the FK-index has not previously been validated outside the USA. Our results demonstrate the predictive ability of the FK-index in a Norwegian nursing home population, with similar test characteristics compared to earlier studies from the USA. ${ }^{[7,9]}$ In our test population, C-index for the proBNP-adjusted index was 0.66, compared to 0.69 in the US validation study, ${ }^{[9]}$ and 0.71 in the index developmental population. ${ }^{[7]}$

Compared to previous test populations, our nursing home population was older and had a higher proportion of patients with dementia. This likely accounts for the higher mortality rate in our material. Annual mortality in our study population was high also compared to the average mortality in long-term nursing homes in Bergen during 2018, which may explain the high FK scores. In the original material, the lowest score (0-2) carried a one-year mortality risk of $7 \%$ as opposed to $34 \%$ in our study (using the original test). ${ }^{[7]}$ Twenty years have passed since Flacker et al. published their study, during which the morbidity and mortality of nursing home patients has increased around the world. ${ }^{[22]}$ For example, a one-year mortality of $28.8 \%$ and $34 \%$ was reported from nursing homes in Iceland in 2010, and Italy in 2020, respectively. ${ }^{[23,24]}$ The higher age and frailty of our study population compared to other long-term residents in Bergen may indicate a selection bias, also contributing to a higher mortality rate in our material compared to the original study. A higher number of study-participants could have strengthened the study, and the external validity is limited due to inclusion of residents from only one municipality.

The regression analysis showed that only a few test parameters (sex, function of daily living, shortness of breath and ProBNP) significantly contribute to the test scores-values in our population, while unexpectedly, several parameters regarding nutritional status were not, in addition to clinical heart failure and age. This finding may point to selection bias, or the need for a larger study to explore these individual parameters. However, for the purposes of our aim, this study shows that the inclusion of NT-ProBNP may improve the discriminatory power of the FK-index. NT-ProBNP is a predictor of mortality independent of heart failure, and was routinely collected in half-yearly medication reviews in our study population. The improved prognostic ability by adding NT-ProBNP to the FK-score, confirms this marker as a strong predictor of death connected to HF compared to the average used methods in the original study. While a heart failure diagnosis may be present with normal ProBNP values, we believe the ProBNP value has additional relevance with respect to mortality estimates as an independent predictor of mortality.

One practical purpose of the FK-index could be to adjust populations for patient-related mortality risk when comparing annual mortality-rates between institutions. Another purpose of the FK index could be to aid medication reviews and advance care planning (ACP). The limited life expectancy of all nursing home residents imply that ACP and medication reviews should be conducted routinely for all nursing home residents. ${ }^{[25,26]}$ However, such reviews and discussions could benefit from identifying residents for whom the main emphasis should be on tapering preventive treatment, symptom relief and individualized care. However, with a C-index of 0.66 we cannot expect a firm one-year mortality prediction even by the use of an NT-ProBNP enhanced FK-index. Endof-life communication with the residents and their families must still convey the uncertainty of prognostic predictions.

\section{Conclusion}

Our study confirms the FK-index ability to predict one-year mortality in a Norwegian nursing home population, with test performance comparable to earlier studies. The inclusion of NT-ProBNP as a test parameter might improve the discriminatory power of the FK-index, and clinical decision making for nursing home residents.

\section{ACKNOWLEDgeMENTS}

We would like to thank Jannicke Igland (Core facility for Biostatistics and Data Analysis, University of Bergen) for statistical advice, and are grateful to all doctors and nurses in our participating institutions.

\section{Conflicts of InTERest Disclosure}

The authors have not reported any competing interests. 


\section{REFERENCES}

[1] Thomas JM, Cooney LM, Jr. Fried TR. Systematic review: Healthrelated characteristics of elderly hospitalized adults and nursing home residents associated with short-term mortality. Journal of the American Geriatrics Society. 2013; 61: 902-11. PMid:23692412 https://doi.org/10.1111/jgs.12273

[2] www.cms.gov. Minimum-Data-Set-3-0-Public-Reports. US: gov; 2020.

[3] Thomas KS, Ogarek JA, Teno JM, et al. Development and Validation of the Nursing Home Minimum Data Set 3.0 Mortality Risk Score (MRS3). J Gerontol A Biol Sci Med Sci. 2019; 74: 219-25. PMid:29514187 https : //doi.org/10.1093/gerona/gly044

[4] Ogarek JA, McCreedy EM, Thomas KS, et al. Minimum Data Set Changes in Health, End-Stage Disease and Symptoms and Signs Scale: A Revised Measure to Predict Mortality in Nursing Home Residents. Journal of the American Geriatrics Society. 2018; 66: 976-81. PMid:29500822 https://doi.org/10.1111/jgs.15305

[5] Yourman LC, Lee SJ, Schonberg MA, et al. Prognostic indices for older adults: a systematic review. JAMA. 2012; 307: 182-92. PMid:22235089 https ://doi .org/10.1001/jama.2011.1966

[6] Flacker JM, Kiely DK. A practical approach to identifying mortalityrelated factors in established long-term care residents. Journal of the American Geriatrics Society. 1998; 46: 1012-5. PMid:9706893 https://doi.org/10.1111/j.1532-5415.1998.tb02759.x

[7] Flacker JM, Kiely DK. Mortality-related factors and 1-year survival in nursing home residents. Journal of the American Geriatrics Society. 2003; 51: 213-21. PMid:12558718 https ://doi.org/10.1046/ j.1532-5415.2003.51060.x

[8] Niznik JD, Zhang S, Mor MK, et al. Adaptation and Initial Validation of Minimum Data Set (MDS) Mortality Risk Index to MDS Version 3.0. Journal of the American Geriatrics Society 2018; 66: 2353-9. PMid:30335184 https://doi.org/10.1111/jgs.15579

[9] Kruse RL, Parker Oliver D, Mehr DR, et al. Using mortality risk scores for long-term prognosis of nursing home residents: caution is recommended. J Gerontol A Biol Sci Med Sci. 2010; 65: 1235-41. PMid:20639529 https : //doi.org/10.1093/gerona/glq120

[10] Pencina MJ, D’Agostino RB, Sr. Larson MG, et al. Predicting the 30-year risk of cardiovascular disease: the framingham heart study. Circulation. 2009; 119: 3078-84. PMid:19506114 https: //doi.org/10.1161/CIRCULATIONAHA.108.816694

[11] Boriani G, Botto GL, Padeletti L, et al. Improving stroke risk stratification using the CHADS2 and CHA2DS2-VASc risk scores in patients with paroxysmal atrial fibrillation by continuous arrhythmia burden monitoring. Stroke: A Journal of Cerebral Circulation. 2011 42: 1768-70. PMid:21493904 https://doi.org/10.1161/STRO KEAHA. 110.609297

[12] Kruse RL, Mehr DR, Petroski GF, et al. Using Mortality Risk Scores for Long-Term Prognosis of Nursing Home Residents: Caution Is Recommended. J Gerontol A Biol Sci Med Sci. 2010; 65(11): 1235 41. PMid:20639529 https ://doi .org/10.1093/gerona/glq12 0
[13] Heckman GA, Hirdes JP, Hebert PC, et al. Predicting Future Health Transitions Among Newly Admitted Nursing Home Residents With Heart Failure. Journal of the American Medical Directors Association. 2019; 20: 438-43. PMid:30573437 https ://doi .org/10.1 016/j.jamda.2018.10.031

[14] Jung M, Yeh AY, Pressler SJ. Heart failure and skilled nursing facilities: review of the literature. Journal of Cardiac Failure. 2012; 18 : 854-71. PMid:23141858 https://doi.org/10.1016/j.cardfa il.2012.09.006

[15] York MK, Gupta DK, Reynolds CF, et al. B-Type Natriuretic Peptide Levels and Mortality in Patients With and Without Heart Failure. J Am Coll Cardiol. 2018; 71: 2079-88. PMid:29747827 https ://doi.org/10.1016/j.jacc.2018.02.071

[16] Barents M, Hillege HH, van der Horst IC, et al. BNP and NT-proBNP, predictors of 1-year mortality in nursing home residents. Journal of the American Medical Directors Association. 2008; 9: 580-5. PMid:19083292 https : //doi .org/10.1016/j.jamda.2008.0 5.002

[17] StatisticsNorway. Oslo. 2019.

[18] Vossius C, Selbaek G, Saltyte Benth J, et al. Mortality in nursing home residents: A longitudinal study over three years. PloS One. 2018; 13: e0203480. PMid:30226850 https ://doi.org/10.137 1/journal.pone. 0203480

[19] Cox DR. Regression Models and Life-Tables. Journal of the Royal Statistical Society Series B (Methodological) 1972; 34: 187-220. https ://doi.org/10.1111/j.2517-6161.1972.tb00899.x

[20] Cochran W. Some methods for strengthening the common chisquared tests. Biometrics International Biometric Society. 1954; 10: 417-51. https://doi .org/10.2307/3001616

[21] Armitage P. Tests for Linear Trends in Proportions and Frequencies. Biometrics International Biometric Society. 1955; 11: 375-86. https://doi.org/10.2307/3001775

[22] JR. Eldres bruk av helse-og omsorgstjenester. Oslo: Statistic Norway; 2013.

[23] Hjaltadottir I, Hallberg IR, Ekwall AK, et al. Predicting mortality of residents at admission to nursing home: a longitudinal cohort study. BMC Health Services Research. 2011; 11: 86. PMid:21507213 https : //doi.org/10.1186/1472-6963-11-86

[24] Braggion M, Pellizzari M, Basso C, et al. Overall mortality and causes of death in newly admitted nursing home residents. Aging Clinical and Experimental Research. 2020; 32: 275-80. PMid:31894560 https : //doi.org/10.1007/s40520-019-01441-x

[25] Martin RS, Hayes B, Gregorevic K, et al. The Effects of Advance Care Planning Interventions on Nursing Home Residents: A Systematic Review. Journal of the American Medical Directors Association. 2016; 17: 284-93. PMid:26861748 https ://doi.org/10.1016/ j.jamda. 2015.12.017

[26] Lawrence S, Robinson A, Eagar K. Identification of the trajectory of functional decline for advance care planning in a nursing home population. Australas J Ageing. 2017; 36: E14-E20. PMid:28925098 https : //doi.org/10.1111/ajag. 12454 